\title{
The Impact of Alerting Design on Air Traffic Controllers' Response to Conflict Detection and Resolution
}

\author{
Peter Kearney ${ }^{1}$, Wen-Chin $\mathrm{Li}^{2}$ and John J. H. Lin ${ }^{3}$ \\ ${ }^{1}$ ATM Operations and Strategy, Irish Aviation Authority, The Times Building, 11-12 D'olier Street, \\ Dublin, Ireland \\ ${ }^{2}$ Safety and Accident Investigation Centre, Cranfield University, Cranfield, Bedfordshire, \\ MK43 0TR, United Kingdom \\ ${ }^{3}$ Humanities and Technology Lab, Lund University, Helgonabacken 12, Sweden
}

Running Head: Alerting Designs Effect Air Traffic Controllers' Conflict Detection

Manuscript Type: Research Article

Exact Word Count of Text: 4,689 words

Abstract: 253 words

References: 40

\section{Biographies}

Peter Kearney

Director of ATM Operations and Strategy, Irish Aviation Authority, Dublin, Ireland

MSc of Safety and Human Factors in Aviation, School of Aerospace, Transport and Manufacturing,

Cranfield University, United Kingdom

Wen-Chin Li PhD C.ErgHF

Senior Lecturer, Safety and Accident Investigation Centre, Cranfield University, United Kingdom $\mathrm{PhD}$ in Aviation Human Factors, College of Aeronautics, Cranfield University, United Kingdom

John Lin PhD

Visiting researcher of Humanities and Technology Lab, Lund University, Helgonabacken 12, Sweden $\mathrm{PhD}$ in Educational Psychology, Institution of Education, National Chiao Tung University, Taiwan

\section{Corresponding author:}

Wen-Chin Li

Martell House, University Way

Cranfield, Bedfordshire

MK43 0TR, England

Telephone: +44 (0) 1234758527

Fax: + 44 (0) 1234752940

Email: wenchin.li@cranfield.ac.uk 


\begin{abstract}
Purposes: The research aim is to develop a better design of auditory alerts that can improve air traffic controllers' situation awareness.

Method: Participants are seventy-seven qualified Air Traffic Controllers. The experiment was conducted in the Air Traffic Control operational rooms of the Irish Aviation Authority at Shannon and Dublin. Participants were advised that the trials were in relation to the COOPANS Air Traffic Control. ANOVA with two between-subject factors (alerting designs and experience levels) were conducted to analyze the ATCO's response time for three critical events. Bonferroni test was performed for post-hoc analysis on mean differences of response time.

Results: There is a significant difference in ATCO's response time between acoustic alert and semantic alert across STCA, APW and MSAW. No significant main effect of controllers' experience on ATCO's response time for STCA and APW. Also, there is no significant interaction between alerting design and experience level on ATCO's response time across STCA, APW and MSAW.

Conclusion: The results demonstrated that the acoustic alert deployed within the COOPANS ATM system provides level-1 Situational Awareness to ATCO's compared with an semantic alert which provides not only level1 of situational awareness for perceived alerts, but also level-2 and level-3 of situational awareness to assist ATCO understanding of critical events and therefore develop more suitable solutions. Consequently, humancentered design of a semantic alert can significantly speed up ATCO's response to STCA, and APW. Furthermore, the sematic alert could alleviate expertise differences by promoting quicker response times for both novice and experienced air traffic controllers.
\end{abstract}

Keywords: Air Traffic Management, Alerting Design, Human-Centered Design, Semantic Alert, Situation Awareness

\title{
1 Introduction
}

The COOPANS Air Traffic Management (ATM) System provides three kinds of alerts which are designed to alert controllers to three distinct critical risks, Short Term Conflict Alert (STCA), Minimum Safe Altitude Warning (MSAW), and Area Proximity Warning (APW). Activation of any of these three alerts by acoustic design indicates a potential conflict of aircraft, conflict between aircraft and prohibited airspace and conflict between aircraft and terrain, therefore the air traffic controller is expected to respond and resolve the potential conflict as fast as possible to ensure the safety of the aircraft. The COOPANS system is deployed in five countries within Europe, Ireland, Denmark, Sweden, Austria and Croatia, and the air traffic controllers (ATCO) within these five countries all operate a harmonized system which provides three critical alerts using the same audio alerting schema. Furthermore, the COOPANS system is seen as a major step in achieving the European Union aspiration of harmonized ATM systems across Europe in support of Single European Sky (Eurocontrol, 2015).

The activation of the STCA alert on the COOPANS system provides a 90 second warning, that unless appropriate action is taken by air traffic controllers to resolve the conflict, significant risk of collision between aircrafts exists. This introduces time pressure on the ATCO's to identify the nature of the alerts and respond with air traffic control instructions which resolve the conflict situation (Eurocontrol, 2014). However, previous safety recommendations from aircraft accidents linked to controlled flight into terrain and aircraft collision revealed that most of these accidents occurred in circumstances where MSAW and STCA were available to ATCO's. All of these accidents demonstrated that alerting system configurations provided alerts of the impending situation, but ATCO's failed to provide timely safety advice to flight crew (NTSB, 2006). The report also indicated that ATM systems were not adequately designed so as to provide ATCO's with effective situation awareness (SA) to make timely decisions. Air traffic controllers must make a rapid judgment of the situation that is being signaled by the automatic alerts provided by an ATM system, and then take the appropriate decision to ensure aviation safety. Interestingly research spanning from 1977 to 2008 has demonstrated that decision errors in aviation may be contributing to up to $60 \%$ of all aviation accidents (Jensen \& Benel, 1977; Buch \& Diehl, 1984; Diehl, 1991; Li \& Harris, 2008). Furthermore, recent accident investigations found that poor air traffic control might impact on aviation safety (Daramola, 2014). The current ATM system is reactive and inefficiencies result in delays with associated negative impacts on economy and safety. Therefore, there is an urgent need to develop novel and strategic methods for ATM, making use of current and future technologies to enable 
better planning and thereby increase capacity and efficiency, without jeopardizing safety (Schuster \& Ochieng, 2014).

\subsection{Alerting designs and ATCO's' situation awareness}

Current COOPANS system has a potential design issue which is an alert activation for STCA, MSAW or APW might be misinterpreted as the acoustic signal for all three alerts is the same (Beep-Beep-Beep-Beep). This has the potential to induce an ATCO's into misjudging the type of critical alert being presented and in the worst circumstances the ATCO's response may be to simply silence the acoustic alert, not solve the separation risk for example, thereby weakening system safety. Design of salient alerts for ATM systems might be excellent at capturing ATCO's attention and increasing SA. However, this salient alert might immediately divert operator's attentional resources away from the ongoing task, incurring other issues such as startle and primary task error by distraction (Imbert, Hodgetts, Parise, Vachon, Dehais \& Tremblay, 2014). Suitably human-centered designs of automated alerts in ATC can have significant effects on controllers' performance and reduced cognitive workload (Laois \& Giannacourou, 1995; Tobaruela, Schuster, Majumdar, Ochieng, Martinez, \& Hendrickx, 2014), with increased capability to perform complex task management (Wickens and Hollands, 2000). However, inappropriate design of automation can present many disadvantages and create potential risk leading to accident/incidents, including loss of SA, and substituting the human operators outside system control loop (Durso, Truitt, Hackworth, Crutchfield, \& Manning, 1998; Endsley, 1995). Intensity of audio alert designs can be modified based on task demands and working environment in order to optimize ATCO's performance. In addition to audio intensity level, the semantic content of an auditory alert that conveys the appropriate hazard level is the central component to increasing operators' SA (Edworthy \& Hellier, 2006). Appropriate design of automation in ATM systems can assist in moderating ATCO workload and improving SA by facilitating a better match between task demand and cognitive resource (Kaber, Perry, Segall, McClernon, and Prinzell, 2006). The effective coordination of the human-automation team is crucial to the successful implementation of ATM systems in the future. Designing and managing human-automation teams require an understanding of principles of cognitive system engineering, allocation of function and team adaptation. It is a holistic approach by distributed cognition coordination to rapidly changing situations (Langan-Fox, Canty, \& Sankey, 2009). Future human-centered design of ATM system shall be based on a strategic, collaborative and automated concept of operations, as high performance in conflict detection and resolution has the potential to increase both airspace efficiency and the safety of aviation (Schuster \& Ochieng, 2014). Based on the above literature review, current research will investigate ATCO's SA between the current acoustic alert and new semantic alert developed by the authors. Therefore, the first null hypothesis of current research is " $\mathrm{H}_{0}$ : designs of alert has no significant effect on ATCO's SA"; and the alternative hypothesis is " $\mathrm{H}_{1}$ : design of semantic alert can improve ATCO's SA compared with acoustic alert".

\subsection{Working experience and information processing}

Task performance between novices and experts are different, experts are better at predicting future states than novices (Roth and Woods, 1988; Wickens, 1992). Experts' mental representations are more organized and conceptually richer than novices; as a consequence, expert's decision-making relies on a deep understanding of situations, whereas novices are based on rigid rules (Hoffman, Trafton and Roebber, 2007). In circumstances of time-limited situations, such as ATC, the faster the mental processing of the task the better the performance and the faster the processing is completed, the more time is available for subsequent tasks (Salthouse, 1992). Experienced controllers showed a higher proportion of relative gaze duration and relative fixation duration on relevant areas within the orientation phase than in the other phases. The reason could be that experts spend more time than novices in forming a conceptual understanding of the problem in order to build up a richer and more organized mental representation of the situation (Bruder, Eibfeldt, Maschke \& Hasse, 2014). Research in aviation demonstrated that experienced pilots performed in a superior manner compared with less experienced pilots in a study at recognizing deteriorating weather conditions (Wiegmann, Goh \& O'Hare, 2002). Furthermore, experts and novices differ in response to dynamic situations; experts were considerably more context-dependent in evaluating situations than novices, allowing them to evaluate situations more holistically than novices (Strauch, 2004). The increased experience in the environment should lead to the formation of higher level structure such as schemata or mental models, which can be used to organize the complexity and multiplicity of objects in the environment; it can be observed by experienced controllers generating a cognitive picture more easily and faster than novice controllers (Endsely \& Bolstad, 1994). There is a need to investigate air traffic controller response time between novices and experts, therefore, the second null hypothesis of current research is " $\mathrm{H}_{0}$ : working experience has no significant effect on ATCO's response time"; and the alternative hypothesis is " $\mathrm{H}_{1}$ :experienced ATCO's response time quicker than novice's response time". 


\subsection{Design of alerts and ATCO's experience for conflict resolution}

Monitoring aircraft information is an important part of the ATCO's task. Previous research identified a number of safety concerns with ATM systems, such as lack of uniqueness of alarms, multiple false alarms, alarms being counter intuitive and alarms being annoying and increasing ATCO's workload (Ahlstrom, 2003; Newman \& Allendofer, 2000). In the ATC domain, the activation of a safety alert means that the air traffic controllers must resolve a critical situation, often while under time pressure. Auditory alerts can attract the operator's attention regardless of where visual attention is directed, if presented at an audio level to sufficiently overcome background noise. However, the effect of auditory alerts can be diminished if the presentation of an acoustic alert is too high which can lead to controllers being startled. Verbal warnings tailored to specific hazard situations may improve hazard-matching capabilities without substantial trade-off in perceived annoyance (Baldwin, 2011). The main purpose of ATC is to optimize the flow of traffic and to separate aircraft to prevent collisions both in the air and on the ground; therefore an examination of all possibilities to enhance ATCO's monitoring performance using advanced technology is required (Alonso et al., 2013). A key objective of ATM system design should be to enable the controller to integrate existing information with the evolving traffic state within the airspace under control. The need to evaluate rapid change in a timely fashion is critical, as inappropriate clearances could place aircraft on conflicting paths thereby disrupting the entire traffic management scheme (Wickens, Miller \& Tham, 1996). Problem-solving studies show fundamental differences between novices and experts in how problems are interpreted, what strategies are devised, what information is used, memory for critical information, and speed and accuracy of problem solving. Experts can see underlying causes and have more complex models of problem solving than novices (Larkin, McDermott, Simon, \& Simon, 1980). Therefore, current research is aiming to conduct an investigation into the current alerting design within the COOPANS Eurocat Air Traffic Management System, and to develop better alerting design to assist ATCO's handle safety critical events such as conflict detection and resolution is a safer more efficient manner. Based on the above literature review, current research will investigate the interaction effect between alerting designs (acoustic vs. semantic alert) and working experience (expert vs. novice) on air traffic controller's response time. The third null hypothesis is " $\mathrm{H}_{0}$ : there is no significant interaction effect of alerting design and working experience on ATCO's response time"; and the alternative hypothesis is " $\mathrm{H}_{1}$ : there is significant interaction effect of alerting design and working experience on ATCO' response time".

In the context of the COOPANS system where all three critical alerts are signaled in the same manner, this may delay an ATCO's problem identification phase and consequently delay the development of resolutions for critical events, it may also have a negative impact on ATCO's performance in conflict detection and resolution (Schuster \& Ochieng, 2014). In order to predict motion and project aircraft position into the future, aircraft moving forward must be used as basis for a cognitive projection model, and this implied that longer and more frequencies of fixations were devoted to processing all relevant information. Although good performance was maintained in conflict trails with respect to no conflict trials, greater workload was experienced and measured (Marchitto, Benedetto, Baccino, \& Canas, 2016). Alert activations are known to have a negative effect on the primary task, through the startle effect, which is manifested by increased workload, prolonged time for decision making, and reduced situation awareness all of which can contribute to human error. (Baile, Konstan, and Carlis, 2001; Imbert, Hodgetts, Vachon, \& Tremblay, 2014). Design of alerting systems require designers to balance the prominence of the alert and the ease of assimilation into the user's primary task (Maglio and Campbell, 2000). Current research is to investigate the effectiveness of the alerting designs within the COOPANS Eurocat System. The research objectives are to capture best practice for alerting design (acoustic design vs. semantic design) through controllers' response time to critical events; to evaluate the conflict resolution time between different experience levels of controllers (expert vs. novice); and to investigate the interaction effects between alerting design and controllers' experience on response time for resolving the potential conflicts.

\section{Method}

\subsection{Participants}

Seventy-seven participants all currently rated Air Traffic Controllers from Irish Aviation Authority, consisting of fifty-eight males and nineteen females took part in the experiment. The approval of Science and Engineering Research Ethics Committee of Cranfield University was granted in advance of the research taking place. All collected data was only available to the research team and was stored in accordance to the University's Ethical Code and the Data Protection Act. 


\subsection{Apparatus}

Training Simulator: The Irish Aviation Authority's contingency and validation platform was used to develop the scenarios for STCA, MSAW and APW alerts for current research. This training platform is an exact copy of the COOPANS Air Traffic Management System. The platform was supplied by THALES Air Traffic Management Systems as part of the COOPANS programme (figure 1a). The software version used was known as THALESB2.1. No adjustment was made to the configuration of acoustic alert presentation within the COOPANS system, the audio alert (Beep-Beep-Beep-Beep) is referred to as the current acoustic alerts for STCA, APW and MSAW. In addition, a semantic alert presentation using a text to speech program used to convert the specific alert nature to spoken word was developed. In this trial a female voice, "Crystal", in English was used as the computer voice for 3 scenarios, such as Beep-Conflict-Conflict-Beep for STCA; Beep-Altitude-Altitude-Beep for MSAW; and Beep-Airspace-Airspace-Beep for APW. The durations of both semantic alerts and acoustic alerts are the same, as ATCOs' response time was measured relative to the onset of the alert.

\subsection{Scenarios Presented}

In order to provide participants with a high fidelity setting, simulated traffic scenarios were developed using the COOPANS training simulator. The trials were faithful to normal operational configuration, all systems were shown to be operating normally, there was no degradation of weather conditions, and all of the aircraft within the training exercises were airlines and aircraft common to Irish airspace. Current Air Traffic Control Training exercises were used to produce events which would trigger the activation of the COOPANS system safety alerts of STCA, MSAW and APW.
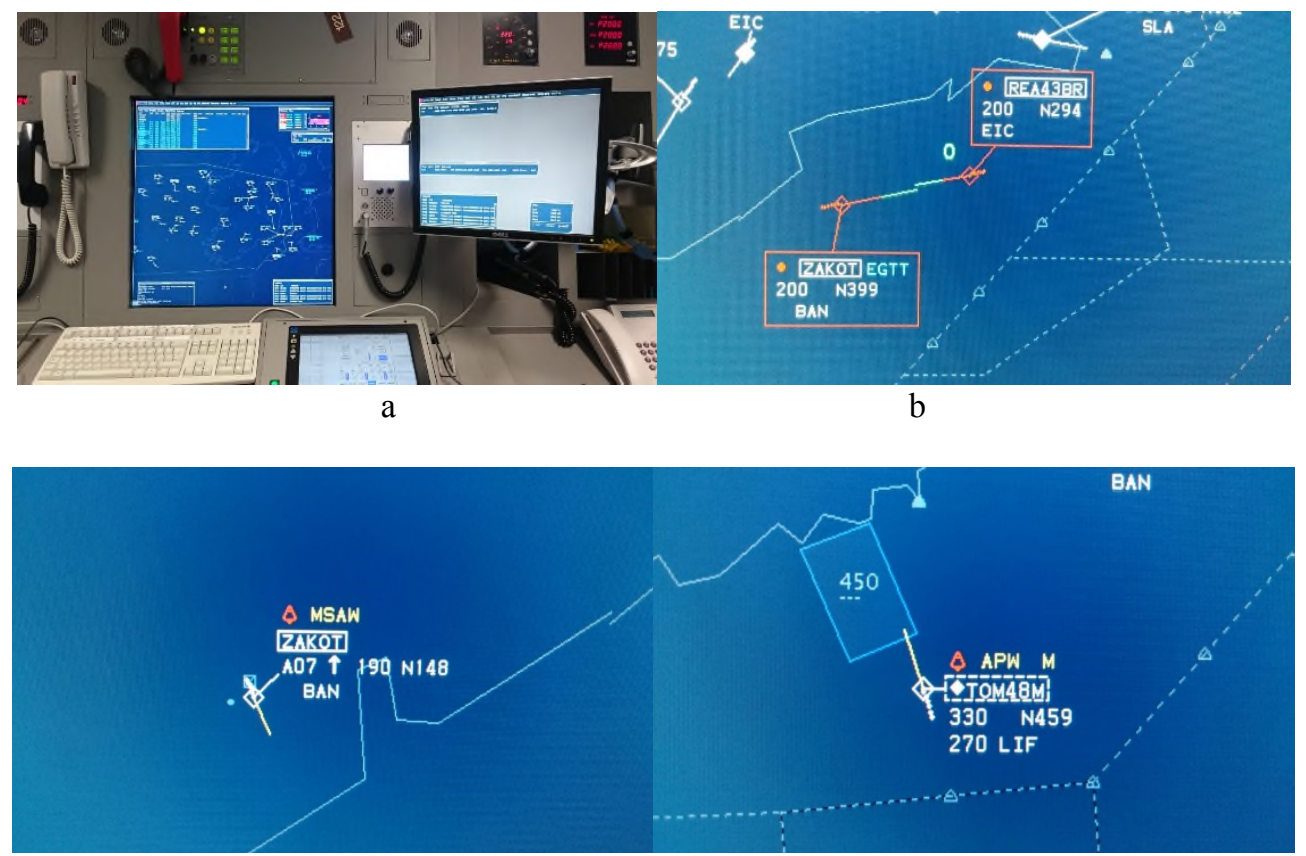

c

d

Figure 1 Examples of the critical events displayed on COOPANS Eurocat Air Traffic Management system (a-d). Note: $\mathrm{a}=$ Air Traffic Controller Working Position, $\mathrm{b}=$ Activated STCA Alert, $\mathrm{c}=$ Activated MSAW Alert, $\mathrm{d}=$ Activated APW Alert

\subsubsection{Short Term Conflict Alert (STCA)}

The airspace sector used represented an approach sector, with the radar range set to $40 \mathrm{~nm}$ and traffic arriving to and departing from an aerodrome in the centre of the display. A total of 18 aircraft were present in the airspace sector displayed. The STCA is to anticipate positional conflicts within a given airspace and to generate warnings for all eligible system track pairs whose separation is expected to be lower than the defined minimum separation requirement of $1,000 \mathrm{ft}$ vertical separation and three nautical miles lateral separation. A pair of tracks in conflict means that the vertical and horizontal separations are infringed. The visual representation of the STCA is shown as figure $1 b$.

\subsubsection{Minimum Safe Altitude Warning (MSAW)}


The airspace sector used represented an approach sector, with the radar range set to $40 \mathrm{~nm}$ and traffic arriving to and departing from an aerodrome in the centre of the display. A total of 18 aircraft were present in the airspace sector were displayed. This alert is intended to alert when any aircraft is infringing or predicted to infringe the relief or minimum sector altitude. It is also designed to predict if an aircraft is projected to deviate from the approach path of an airport. The visual representation of the MSAW is shown as figure 1c.

\subsubsection{Area Proximity Warning (APW)}

The airspace sector used represented an approach sector, with the radar range set to $40 \mathrm{~nm}$ and traffic arriving to and departing from an aerodrome in the centre of the display. A total of 18 aircraft were present in the airspace sector were displayed. This alert provides a warning that an aircraft is foreseen to enter a Military Operating Area, Danger Area or Prohibited area. The visual representation of the APW is shown as figure 1d.

\section{$2.4 \quad$ Research Design}

Participants were advised that the trials were in relation to operating the COOPANS Air Traffic Control System and were of approximate 30 minutes duration. Participants were advised that on hearing the audio alert sound they should mark on the radar screen shot the aircraft or aircrafts that were creating the alerting event. The commencement of each trial would be announced by the facilitator. When it was confirmed that the participant understood the processes of the trial, the experiments commenced. Each participant completed three scenarios related to radar screen shots of STCA, APW, and MSAW. Simultaneously, once the participant advised they were ready to begin, the appropriate audio sound (either current COOPANS acoustic alert or Semantic Alert) was activated on Toshiba Portege R830-138. Activation of the recording time was synchronized with the activation of the alert, and the response time was recorded upon correct response to the alert. This process continued until all three scenarios of radar screen shots had been completed. Each participant encountered occurrences of STCA, APW, and MSAW randomly in order to eliminate practice effects. The audio alert presented to the Air Traffic Controllers in trial-A is the acoustic alert that is available within the COOPANS system (Beep-BeepBeep-Beep). The semantic alert of Trial-B consisted of a new semantic audio alert (Beep-Conflict-ConflictBeep). A three-way mixed-design ANOVA with ATCO's response time as the dependent variable was conducted. Alerting design (acoustic alert vs. semantic alert) and experience level (novice vs. expert) were two between-subject factors. Considering the generalizability, two designs were assessed in three scenarios (STCA, APW \& MSAW). Scenarios were the within-subject factor. A full factorial design was implemented while evaluating interactions among factors. The adjusted degree of freedom was based on the result of Mauchly's test. Significant level was set at $\alpha=.05$ for all analysis. Bonferroni tests were performed to identify pairwise differences for factors with more than two levels. Partial eta-square $\left(\eta^{2}\right)$ is a measure of effect size for ANOVA, whereas Cohen's d is an effect size used to evaluate the standardized difference between two means in this study.

\section{$3 \quad$ Results}

\subsection{Sample Characteristics}

Seventy-seven Air Traffic Controllers participated in the current research, thirty-eight participants completed trials using the current COOPANS acoustic alerts, and thirty-nine participants completed the trials using the semantic alert. Participants' working experience as rated air traffic controllers were between 1 and 40 years (M $=11.71, \mathrm{SD}=8.58)$. Thirty-five participants had worked as Air Traffic Controllers for less than ten years and were classified as novices; forty-two participants had worked as Air Traffic Controllers for more than ten years and were classified as experts. Age of the participants ranged from 20 years old to 62 years old $(\mathrm{M}=36.69, \mathrm{SD}$ = 8.79). Response time (seconds) of STCA, APW \& MSAW scenarios were collected. Descriptive statistics were showed in table 1.

Table 1. ATCO's means (standard deviations) response time of conflict resolution by alerting designs and working experience across three critical scenarios

\begin{tabular}{lllclc}
\hline $\begin{array}{l}\text { Alerting } \\
\text { design }\end{array}$ & $\begin{array}{c}\text { Experienced } \\
\text { levels }\end{array}$ & $n$ & STCA & APW & MSAW \\
\hline Acoustic & Novice & 16 & $4.87(2.47)$ & $3.13(0.86)$ & $3.24(1.05)$ \\
& Expert & 22 & $5.41(3.67)$ & $3.06(0.98)$ & $4.06(3.42)$
\end{tabular}




\begin{tabular}{llllll} 
Semantic & Novice & 19 & $2.46(0.64)$ & $2.60(0.76)$ & $2.46(0.73)$ \\
& Expert & 20 & $2.65(0.70)$ & $2.75(0.77)$ & $3.09(1.07)$ \\
\multirow{3}{*}{ Total } & Novice & 35 & $3.56(2.10)$ & $2.84(0.84)$ & $2.81(0.96)$ \\
& Expert & 42 & $4.10(3.00)$ & $2.91(0.89)$ & $3.59(2.60)$ \\
\hline
\end{tabular}

\subsection{Testing main effects and interactions among factors on air traffic controllers' response time}

Table 2 summarized the result of three-way ANOVA for alerting design, experience, and scenarios. Based on the result of Mauchly's test, the assumption of sphericity was not violated, $\chi^{2}=5.52, p=.063$. The results indicated that no three-factor interaction (alerting designs $\times$ experience levels $\times$ scenarios), $\mathrm{F}(2,146)=0.196, p$ $>.05$. No significant interaction between working experience and scenarios, $\mathrm{F}(2,146)=0.956, p=.39$. No significant interaction between alerting designs and working experience, $\mathrm{F}(1,73)=0.032, p=.86$. Therefore, the third null hypothesis $\left(\mathrm{H}_{0}\right.$ : the interaction of alerting designs and working experience would have no effect on ATCO's response time) was not rejected. In summary, the design of the semantic alert significantly reduced ATCO's response time for both expert and novice (figure 2).

Table 2. Three-way analysis of variance for alerting design, experience, and scenario

\begin{tabular}{lrrrrrr}
\hline \multicolumn{1}{c}{ Source } & $\mathrm{df}$ & $\mathrm{SS}$ & $\mathrm{MS}$ & $\mathrm{F}$ & $p$ & $\eta^{2}$ \\
\hline Between-subject factor & & & & & & \\
Design & 1 & 31.7 & 31.7 & 17.3 & $<$ & 0.192 \\
& & 13 & 13 & 56 & .001 & \\
Experience & 1 & 2.68 & 2.68 & 1.46 & 0.23 & 0.020 \\
& & 0 & 0 & 7 & 0 & \\
Design $\times$ Experience & 1 & 0.05 & 0.05 & 0.03 & 0.85 & $<.001$ \\
& & 9 & 9 & 2 & 7 & \\
Residual & 73 & 133. & 1.82 & & & \\
& & 387 & 7 & & & \\
Within-subject factor & & & & & & \\
Scenario & 2 & 36.5 & 18.2 & 7.89 & $<$ & 0.098 \\
& & 25 & 63 & 2 & .001 & \\
Design $\times$ Scenario & 2 & 49.1 & 24.5 & 10.6 & $<$ & 0.127 \\
Experience $\times$ Scenario & & 80 & 90 & 26 & .001 & \\
& 2 & 4.42 & 2.21 & 0.95 & 0.38 & 0.013 \\
Design $\times$ Experience $\times$ Scenario & & 4 & 2 & 6 & 7 & \\
Residual & 2 & 0.78 & 0.39 & 0.16 & 0.84 & 0.002 \\
& & 4 & 2 & 9 & 4 & \\
\hline
\end{tabular}

Note: $d f$ : degree of freedom, SS: sum of square, MS: mean square, $\eta^{2}$ : partial eta square

\section{STCA}

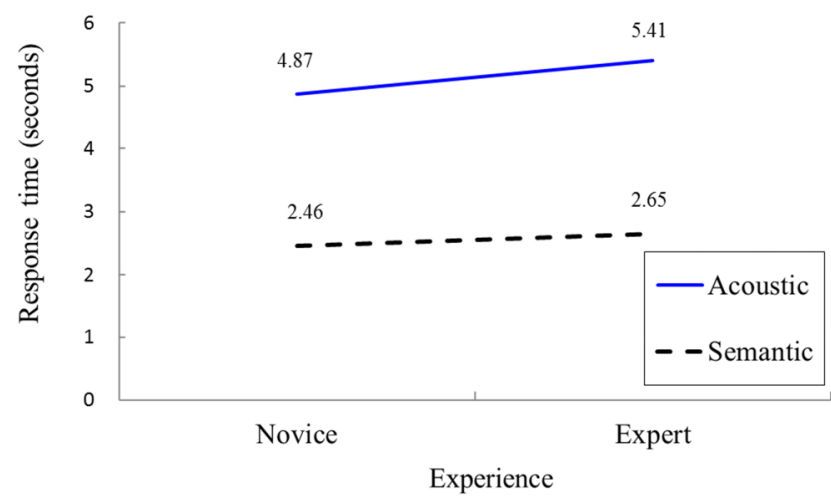


APW

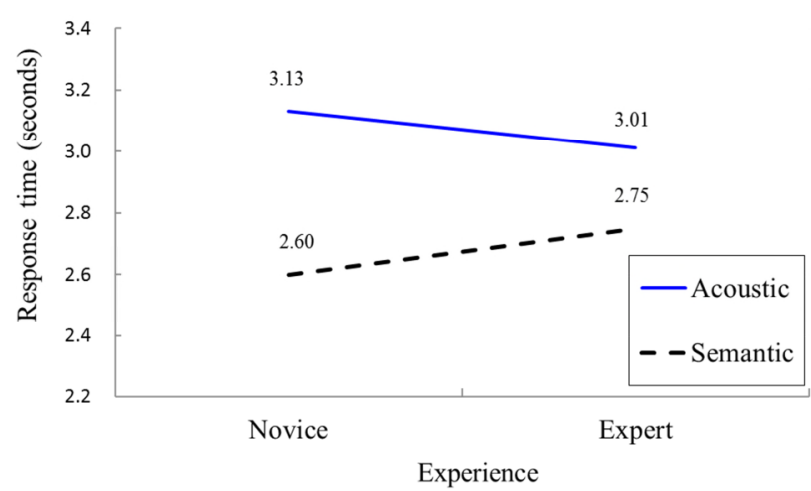

MSAW

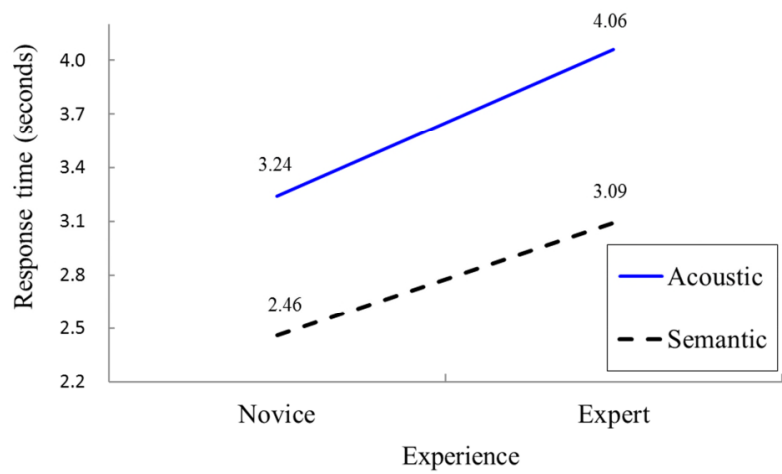

Figure 2. For both expert and novice, ATCO's response time significant reduce by semantic alert compared with acoustic alert on STCA, MSAW, APW, respectively.

However, a significant interaction between alerting design and scenario was revealed, $\mathrm{F}(2,146)=10.626, p$ $<.001, \eta^{2}=.127$. A significant simple main effect for the design within the STCA scenario revealed ATCOs required more time in response to acoustic alert $(5.182 \mathrm{~s})$ than semantic alert $(2.562 \mathrm{~s}), \mathrm{F}(1,75)=25.142, p$ $<.001, \eta^{2}=.251$. Also, a significant simple main effect for the design within the APW scenario revealed ATCOs required more time in response to acoustic alert (3.091s) than semantic alert $(2.677 \mathrm{~s}), \mathrm{F}(1,75)=4.662$, $p<.05, \eta^{2}=.059$. A significant simple main effect for the design within the MSAW scenario revealed ATCOs required more time in response to acoustic alert (3.712s) than semantic alert $(2.779 \mathrm{~s}), \mathrm{F}(1,75)=4.124, p<.05$, $\eta^{2}=.052$. In summary, the semantic alert is promising in efficiently conveying crucial message to ATCOs (figure 3)

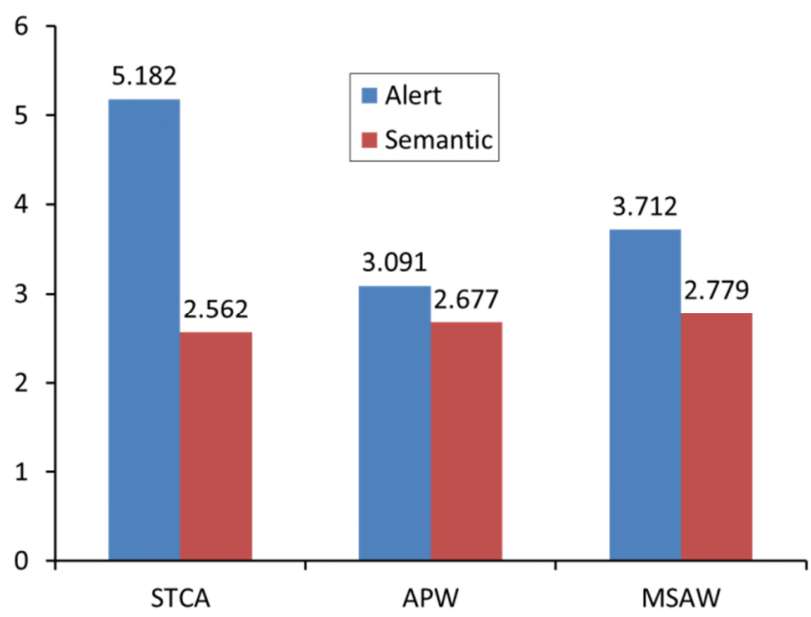

Figure 3 Comparison of ATCO's response time among two alert design within three scenarios.

For the alerting design, a significant main effect was found, $\mathrm{F}(1,73)=17.356, p<.001, \eta^{2}=.192$. Air traffic controllers required more time in response to acoustic alert (3.961s) than semantic alert (2.669s). Therefore, the 
first null hypothesis $\left(\mathrm{H}_{0}\right.$ : Designs of alert would have no effect on ATCO's response time) was rejected. The main effect of experience is not significant, $\mathrm{F}(1,73)=1.476, p>.05$. Therefore, the second null hypothesis (H0: Working experience would have no effect on ATCO's response time) was not rejected. The scenario did have a main effect on ATCO's response time, $\mathrm{F}(2,146)=7.892, p<.001, \eta^{2}=.098$. The results of Bonferroni posthoc comparison indicated that the mean response time for STCA $(3.849 \mathrm{~s})$ was significantly longer than that in APW (2.886s), $\mathrm{t}=3.733$, adjusted $p<.001$. There was also a trend that the response time for STCA (3.849s) was longer than MSAW (3.209s), $\mathrm{t}=2.397$, adjusted $\mathrm{p}=.05$.

\section{Discussion}

In the COOPANS Air Traffic Management System where all three critical alerts are signaled in the same manner "Beep-Beep-Beep-Beep", activated alerts may confuse problem identification and consequently delay ATCO's problem-solving strategies to deal with different critical events. Strauch (2004) proposed that high workload, competing task demands, and ambiguous cues can all contribute to an operator's loss of SA, even with experienced and well-trained operators. A key prerequisite of conflict detection and resolution is the capability to guarantee situation awareness amongst relevant stakeholders in order to optimize safe air traffic flows (Schuster \& Ochieng, 2014). ATM specialists have clearly defined situation assessment as the process by which the state of situation awareness is achieved (Sarter \& Woods, 1994). There is a need to improve air traffic controllers' situational awareness by designing human-centered alerts in response to critical events.

\subsection{The design of semantic Alert Significant reducing ATCO's response time}

Based on statistical analysis, there are significant differences in ATCO response time between acoustic alert and semantic alert consistently across STCA, and APW (table 1). The current COOPANS acoustic alert only provides an auditory stimulus by an activated "Beep" to signal "something critical happening", thereafter ATCO's have to figure out what is causing the critical situation and identify exactly what alert was activated among STCA, or APW in order to develop an appropriate resolution strategy. All of these processes present a risk that an incorrect judgment of the type of alert being signaled could result in the application of an inappropriate resolution strategy. Therefore, the acoustic alert only provides level-1 SA for ATCO's, i.e. they perceive the activated critical event (Endsley, 1995). Despite, high intensity auditory alerts can draw the operator's attention, the alerting effect can also be annoying and lead to controllers being startled thereby further delaying efficient responses (Marshall, Lee, \& Austria, 2007). A poorly designed acoustic alert can distract the operator, decrease situation awareness and subsequent continuous signaling of the alarm can not only disrupt the resolution of the critical situation but also reduce the operator's ability to manage workload (Banbury, Macken, Tremblay \& Jones, 2001). By contrast, the newly designed semantic alert provides ATCO's with an initial BEEP to signal "I have an important message for you", following by provided the nature of the alert, i.e. CONFLICT, AIRSPACE or ALTITUDE. The ATCO's can no longer be startled by the activation of the audio warning and as the enhanced design of alert is now more semantically rich and is informing the ATCO precisely the nature of the critical problem, no further cognitive load of evaluating the characteristics of the current situation is required, the ATCO can immediately begin to develop resolution strategies suitable to the specific critical event, thereby providing crucial extra time in a time-limited situation to deploy the most appropriate resolution response. Although solid evidence shows that semantic alert outperforms the acoustic alert across three scenarios, based on the effect size (partial eta square), we did observe that the semantic alert has a better / more efficient effect in STCA $\left(\eta^{2}=.251\right)$ than that in either APW $\left(\eta^{2}=.059\right)$ or MSAW $\left(\eta^{2}=.052\right)$. Given our experiment was not counterbalanced, we cannot exclude the possibility that the ordinal effect (period effect) exists. Another possibility is that the three scenarios did not occur evenly (roughly the occurrences of STCA, APW and MSAW are $92 \%, 6 \%$, and $2 \%$ respectively), therefore the rates of occurrences might have an impact on the effect of semantic alert across three scenarios.

\subsection{Human-center design improving ATCO's situation awareness}

The results demonstrate that the semantic alert has improved ATCO's situational awareness as a result of a more meaningful salient alert. The design of the semantic alert significantly decreased ATCO's response time by not only presenting salient signals (perceive the alert, level-1 of SA), but also providing ATCO's with knowledge of the nature of the situation to be resolved (understanding current situation, level-2 of SA). Therefore, ATCO's have more time to project the evolving flow of aircraft in the near future (projection of future status, level-3 of SA), and develop more effective resolutions to critical events, permitting a more rapid return to ensuring the rest of the aircraft under his/her control continue to be managed in a safe and efficient way (Endsley, 1997). There 
is no significant effect on ATCO response time between novice and experienced controllers consistently across STCA, APW and MSAW (Table 1), suggesting the semantic design outperforms the acoustic design for both novice and expert air traffic controllers. Based on table 1, ATCO's response time on STCA showed the most noticeable difference between acoustic alerts and semantic alerts. This is an interesting finding that may be a difference in the processing resources required for comprehension of alert on each scenario. For instance, the figure (1b) suggest that by default the STCA is an acoustic-only warning that requires the operator understanding the depicted relationship between 2 aircraft, as the STCA screen does not explicitly show "STCA." However, in the other two scenarios (MSAW, figure 1c ; APW, figure 1d), there is an explicit notation of the warning type present on the screen as "MSAW" or "APW," and these types of warnings only involve a single aircraft (not the relationship among 2 aircraft). Because the STCA warning type does not explicitly show the warning type on the screen, and is complicated by having to understand the relationship between 2 aircraft, it may require more cognitive resources toward resolving the nature of the warning. Because of this, the semantic alert might prove most beneficial in this condition. Furthermore, the semantic alert can promote a better understanding of technology-mediated interaction in ATM systems, as it offers specific information across the ATM systems that influence ATCO's cognitive processes from perception to action by providing the representation and projection of air traffic information. The improvement of ATCO's response time is related to the more humancentered design of the semantic alerts, as it provides ATCO's with knowledge of the critical situation occurring. This is achieved by ensuring that the semantic alert is sufficiently salient (initiated by Beep) so that the attentional mechanism and perception process is engaged, followed by the precise nature of critical event (verbal instruction of CONFLICT) reducing the requirement to revert to working memory as there is no necessity to interpret the activated alerts. The results of current research demonstrated that both novice and expert ATCO's response time were significantly reduced in the response to the semantic alert compared with acoustic alert of COOPANS.

\subsection{Semantic design off-loading ATCO's working memory}

The design of the semantic alert has demonstrated a significant main effect of reducing ATCO's response time for both experts and novices across three scenarios. Embedded within many advanced technologies are sophisticated auditory alarms, aimed at drawing the attention of ATCO's to an event of concern. The fundamental issue is that a poorly designed audio alert system has the potential to annoy and distract the operator, to disrupt the tasks that the operator is engaged in and to prolong information processing. All of these have the potential to prevent the operator accurately identifying, prioritizing and responding to abnormal conditions despite the presence of an alerting system designed to enhance safety and assist in retrieving a critical situation (Wood, O'Brien \& Hanes, 1987). The human-centered design of the semantic alert provided to ATCO's in this experiment provided them with knowledge, allowing them to react rapidly and accurately in an information rich environment. Furthermore, the semantic alerts were easily distinguishable from each other. The risk of an operator being induced into an error when systems use the same signal to annunciate a number of different alerts is eliminated (Allendorfer, Friedman-Berg \& Pai, 2007). To summarize, current research results, semantic alerts could alleviate the expertise differences between novice and expert of ATCO's by promoting quicker response time to critical events regardless of working experience. It is obvious ATCO's response time is primarily influenced by the design of the alerting schemata, as the design of semantic alert facilitated ATCO's information processing and provided them with specific knowledge in the form of mental model needed by controllers. This semantic alert is proposed as a method for improving ATCO situation awareness by providing for the integration and comprehension of information and facilitating the projection of future status (the higher levels of SA), thus significantly off-loading ATCO's working memory and efficiently directing cognitive processes.

\section{Conclusion}

Technology development within Air Traffic Management continues at a pace with substantial programs underway in both Europe through Single European Sky ATM Research and in the United States through Next Generation Air Transportation System. Both programs aim to revolutionize air traffic management provision through advanced technologies. Consequently the requirement to provide air traffic controllers with human-centered design of alerts which are appropriately salient and accurate response to critical situations in order to minimize the side-effect of startle should be of primary importance. The results of current research demonstrated that the acoustic alert of COOPANS provides level-1 of SA to ATCO compared with the enhanced semantic alert providing not only level-1 of SA (perception of the alert), but also level-2 of SA assisting ATCO understanding the nature of critical event for developing quick solutions. Consequently, the design of enhanced semantic alert 
can significantly reduce ATCO's response time across STCA, APW and MSAW. The use of semantic alerts as part of the COOPANS Air Traffic Management system saved ATCO's response time by providing the nature of the critical situation, therefore providing valuable extra time in a time-limited situation. Furthermore, semantic design could alleviate the expertise differences between novice and expert of ATCO's by promoting quicker response time to critical events regardless of level of expertise. The findings of current research indicate that a single training program integrated with human-centered design of semantic alert could improve both novice and expert ATCO's situational awareness and significantly reduce response time to critical events. This represents significant benefit to the air navigation service provider to reduce training cost. An increasing in air traffic sector capacity allows improved ATCO productivity, initial estimates put this productivity improvement at $1 \%$ annually. This represents a saving of $€ 2,728,337$ per annum across all five COOPANS Air Navigation Service Provider's or $€ 13,641,685$ over EU SES RPII (Eurocontrol ACE, 2013). The fact that the critical situations are being resolved more safely and efficiently means that ATCO can resume normal operations within the rest of the air traffic control sector sooner. Overall this is foreseen to possibly enable increased capacity within sectors. Civil Aviation Authorities, Air Navigation Service Providers and Air Traffic Management system providers would have benefits from the findings of current research with a view to ensuring that Air Traffic Controllers are provided with the most optimal alerting schemes to increase their situational awareness and performance to handle the unforeseen critical events.

\section{$6 \quad$ Future Application}

The semantic alerting design of the COOPANS Air Traffic Management has the potential to save ATCO's response times to critical situations, providing more time for solution generation and improving the likelihood that the most appropriate resolution strategy will be deployed. The findings of current research provides a means for assessing the benefit of design concepts (acoustic alert vs semantic alert) by the context of the full industrialscale system (COOPANS Air Traffic Management System) and employed trained operators (licensed Air Traffic Controllers) under realistic conditions (STCA, APW and MSAW), creating a rich level of information related to ATCO's performance. From professional perspective this is a valuable finding, as it indicates that a single training program can be developed and provided to both novice and expert ATCO with a result of improving safety performance and quicker response time to critical situations. The applications of current research not only could reduce ATCO's training cost for Air Navigation Service Providers, but also Air Traffic Management System providers would have benefits from ATCO by provided with the most optimal alerting schemes to increase their situational awareness and performance to ensure aviation safety.

\section{Acknowledgements}

Authors would like to express special thanks to the Irish Aviation Authority for their support in funding this research (Ref: 1020470002253004), and also to the air traffic controllers' who took part in the research. Especially the authors would like to thank Declan Mangan, IAA Operations Manager Ballycasey; Paul McCann, IAA Operations Manager Dublin; Peter Kavanagh, IAA Manager Operational Support Group; and Gerald Caffrey, Manager Support Co-ordination. Their supports and the enthusiasm of their respective teams were invaluable in facilitating the authors' research efforts.

\section{References}

1. Ahlstrom, V. (2003). An Initial Survey of National Airspace System Auditory Alarm Issues in Air Traffic Control (DOT/FAA-CT-TN03/10), Federal Aviation Administration, Atlantic City, NJ. Retrieved from http://hf.tc.faa.gov/technotes/dot-faa-ct-tn03-10.pdf

2. Allendorfer, K., Friedman-Berg, F., \& Pai, S. (2007). Human Factors Analysis of Safety Alerts in Air Traffic Control (DOT/FAA/TC-07/22), Federal Aviation Administration, Atlantic City, NJ. Retrieved from http://hf.tc.faa.gov/technotes/dot_faa_tc_07_22.pdf

3. Alonso, R., Causse, M., Vachon, F., Parise, R., Dehais, F., \& Terrier, P. (2013). Evaluation of Head-free Tracking as an Input Device for Air Traffic Control. Ergonomics, 56(2), 246-255. doi: 10.1080/00140139.2012.744473. 
4. Bailey, B. P., Konstan, J. A., \& Carlis, J. V. (2001). The Effects of Interruptions on Task Performance, Annoyance, and Anxiety in the User Interface. In Proceedings of INTERACT (Vol. 1, pp. 593-601). Tokyo, Japan: IDF.

5. Baldwin, C. L. (2011). Verbal Collision Avoidance Messages during Simulated Driving: Perceived Urgency, Alerting Effectiveness and Annoyance. Ergonomics, 54(4), 3285-337. doi:10.1080/00140139.2011.558634

6. Banbury, S. P., Macken, W. J., Tremblay, S., \& Jones, D. M. (2001). Auditory distraction and short-term memory: Phenomena and practical implications. Human factors, 43(1), 12-29. doi:10.1518/001872001775992462

7. Bruder, C., Eibfeldt, H., Maschke, P., \& Hasse, C. (2014). A Model for Future Aviation Operator Monitoring Appropriately. Aviation Psychology and Applied Human Factors, 4(1), 13-22. doi:10.1027/21920923/a000051

8. Buch, G., \& Diehl, A. (1984). An Investigation of the Effectiveness of Pilot Judgement Training. Human factors, 26(5), 557-564. doi:10.1177/001872088402600507

9. Diehl, A. (1991). The Effectiveness of Training Programs for Preventing Aircrew Error, In Jensen, R.S. (Ed.), In Proceedings of the Sixth International Symposium on Aviation Psychology (Vol. 2, pp. 640-655). Colombus, Ohio: The Ohio State University.

10. Daramola, A. Y. (2014). An investigation of air accidents in Nigeria using the Human Factors Analysis and Classification (HFACS) framework. Journal of Air Transport Management, 35, 39-50.

11. Durso, F. T., Truitt, T. R., Hackworth, C. A., Crutchfield, J. M., \& Manning, C. A. (1998). En Route Operational Errors and Situational Awareness. The International Journal of Aviation Psychology, 8(2), 177194. doi:10.1207/s15327108ijap0802_6

12. Edworthy, J., and Hellier, E. (2006). Complex Nonverbal Auditory Signals and Speech Warning. In M. S. Wogalter (Ed.), Handbook of Warnings (pp. 199-220). Mahwah, NJ: Lawrence Erlbaum.

13. Endsley, M. R. (1995). Measurement of Situation Awareness in Dynamic Systems. Human Factors, 37(1), 65-84. doi:10.1518/001872095779049499

14. Endsley, M. R. (1997). The Role of Situation Awareness in Naturalistic Decision Making. In C. E. Zsambok \& G. Klein (Eds.), Naturalistic Decision Making (pp. 269-284), Hillsdale, NJ: Lawrence Erlbaum.

15. Endsley, M. R., \& Bolstad, C. A. (1994). Individual Differences in Pilot Situation Awareness. The International Journal of Aviation Psychology, 4(3), 241-264. doi:10.1207/s15327108ijap0403_3

16. Eurocontrol (2014). Eurocontrol Seven-year IFR Flight Movements and Service Units Forecast: $2014-$ 2020 (Reference No. 14/02/24-43). Brussels, Belgium. Retrieved from www.eurocontrol.int/sites/default/files/content/documents/official-documents/forecasts/seven-yearflights-service-units-forecast-2014-2020-feb2014.pdf

17. Eurocontrol (2015). ATM Cost- Effectiveness (ACE) 2013 Benchmarking Report with 2014-2018 outlook. Brussels, Belgium.

18. Hoffman, R. R., Trafton, G., and Roebber, P. (2007). Minding the Weather: How Expert Forecasters Think. Cambridge, MA: MIT Press.

19. Imbert, J. P., Hodgetts, H. M., Parise, R., Vachon, F., Dehais, F., \& Tremblay, S. (2014). Attention Costs and Failures in Air Traffic Control Notifications. Ergonomics, 57(12), 1817-1832. doi:10.1080/00140139.2014.952680

20. Jensen, R. S., \& Bernel, R.A. (1977). Judgment Evaluation and Instruction in Civil Pilot Training. Washington, D.C.: Federal Aviation Administration.

21. Kaber, D. B., Perry, C. M., Segall, N., McClernon, C. K., \& Prinzell, L. J. (2006). Situation Awareness Implications of Adaptive Automation for Information Processing in an Air Traffic Control-related Task. International Journal of Industrial Ergonomics, 36(5), 447-462. doi:10.1016/j.ergon.2006.01.008

22. Langan-Fox, J., Canty, J. M. \& Sankey, M. J. (2009). Human-automation Teams and Adaptable Control for Future Air Traffic Management. International Journal of Industrial Ergonomics, 39, 894-903. doi:10.1016/j.ergon.2009.04.002

23. Laois, L., \& Giannacourou, M. (1995). Perceived Effects of Advanced ATC Functions on Human Activi- 
ties: Results of a Survey on Controllers and Experts. In Jensen, R.S. (Ed.), In Proceedings of the Eighth International Symposium on Aviation Psychology (Vol. 2, pp. 392-397). Colombus, Ohio: The Ohio State University.

24. Larkin, J. H., McDermott, J., Simon, D. P., \& Simon, H. A. (1980). Expert and Novice Performance in Solving Physics Problems. Science, 208(4450), 1335-1342. doi:10.1126/science.208.4450.1335

25. Li, W-C., \& Harris, D. (2008). The Evaluation of the Effect of a Short Aeronautical Decision-making Training Program for Military Pilots. International Journal of Aviation Psychology, 18(2), 135-152. doi:10.1080/10508410801926715

26. Maglio, P. P., \& Campbell, C. S. (2000). Trade-offs in Displaying Peripheral Information. In Proceedings of the SIGGHI Conference on Human Factors in Computing Systems (pp.241-248). New York, NY: ACM. doi: $10.1145 / 332040.332438$

27. Marchitto, M., Benedetto, S., Baccino, T. \& Canas, J. J. (2016). Air Traffic Control: Ocular Metrics Reflect Cognitive Complexity. International Journal of Industrial Ergonomics, 54, 120-130. doi:10.1016/j.ergon.2016.05.010

28. Marshall, D. C., Lee, J. D., \& Austria, P. A. (2007). Alert for in-vehicle Information System: Annoyance, Urgency, and Appropriateness. Human Factors, 49(1), 145-157. PMID:17315851

29. National Transportation Safety Board (2006). MSAW and Conflict Alert Systems Functionality (Report No. A-06-44-47). Washington, D.C. Retrieved from http://www.ntsb.gov/safety/safetyrecs/RecLetters/A06_44_47.pdf

30. Newman, R. A., \& Allendoerfer, K., (2000). Assessment of current and proposed audio alarms in Terminal Air Traffic Control (DOT/FAA/CT-TN00/21). Federal Aviation Administration, Atlantic City, NJ. Retrieved from http://ntl.bts.gov/lib/30000/30900/30987/TN0021.pdf

31. Roth, E. M., \& Woods, D. D. (1988). Aiding Human Performance I : Cognitive Analysis. Le Travail Humain, 51(1), 39-64.

32. Salthouse, T. A. (1992). Mechanisms of Age-cognition Relations in Adulthood. Hillsdale, NJ: Psychology Press.

33. Sarter, N. B., \& Woods, D. D. (1994). Pilot Interaction with Cockpit Automation II: An Experimental Study of Pilots' Model and Awareness of the Flight Management System. The International Journal of Aviation Psychology, 4(1), 1-28. doi:10.1207/s15327108ijap0401_1

34. Schuster, W. \& Ochieng, W. (2014). Performance requirements of future Trajectory Prediction and Conflict Detection and Resolution tools within SESAR and NextGen: Framework for the derivation and discussion. Journal of Air Transport Management, 35, 92-101.

35. Strauch, B. (2004). Investigation Human Error: Incidents, Accidents, and Complex Systems. Aviation, Space, and Environmental Medicine, 75(4), 372-372.

36. Tobaruela, G., Schuster, W., Majumdar, A., Ochieng, W.Y., Martinez, L., \& Hendrickx, P. (2014). A method to estimate air traffic controller mental workload based on traffic clearances. Journal of Air Transport Management, 39, 59-71.

37. Wickens, C. D. (1992). Workload and situation awareness: An analogy of history and implications. Insight: The Visual Performance Technical Group Newsletter, 14(4), 631-651.

38. Wickens, C. D., \& Holland, J. G., (2000). Engineering Psychology and Human Performance (3rd ed.). Upper Saddle River, NJ: Prentice Hall.

39. Wickens, C. D., Miller, S., \& Tham, M. (1996). The Implications of Data-link for representing Pilot Request Information on 2D and 3D Air Traffic Control Displays. International Journal of Industrial Ergonomics, 18(4), 283-293. doi:10.1016/0169-8141(95)00065-8

40. Wiegmann, D. A., Goh, J., \& O’Hare, D. (2002). The Role of Situation Assessment and Flight Experience in Pilots' Decisions to Continue Visual Flight Rules Flight into Adverse Weather. Human Factors, 44(2), 187-197. doi:10.1518/0018720024497871

41. Woods, D. D., O’Brien, J. F., \& Hanes. L. F. (1987). Human Factors challenges in process control: The case of nuclear Power plants, In G. Salvedy (Ed.), Handbook of Human Factors (pp.1724-17700). Oxford, England: John Wiley \& Sons. 
42. Wiegmann, D. A., Goh, J., \& O'Hare, D. (2002). The Role of Situation Assessment and Flight Experience in Pilots' Decisions to Continue Visual Flight Rules Flight into Adverse Weather. Human Factors, 44(2), 187-197. doi:10.1518/0018720024497871 
2016-09-23

The impact of alerting design on air traffic controllers' response to conflict detection and resolution

\section{Kearney, Peter}

\section{Elsevier}

Peter Kearney, Wen-Chin Li and John J. H. Lin. The impact of alerting design on air traffic controllers' response to conflict detection and resolution. International Journal of Industrial Ergonomics, Volume 56, November 2016, pp.51-58 https://dspace.lib.cranfield.ac.uk/handle/1826/10685 Downloaded from Cranfield Library Services E-Repository 